\title{
On the Toppling of a Sand Pile
}

\author{
Jean-Christophe Novelli ${ }^{1}$ and Dominique Rossin ${ }^{2}$ \\ ${ }^{1}$ CNRS, LIFL, Bâtiment M3, Université Lille 1, F-59655 Villeneuve d'Ascq Cedex, France - Corresponding author \\ ${ }^{2}$ LIX, École Polytechnique, F-91128 Palaiseau Cedex, France
}

received January 30, 2001, revised April 10, 2001, accepted April 12, 2001.

\begin{abstract}
In this paper, we provide the first study of the sand pile model $S P M(0)$ where we assume that all the grains are numbered with a distinct integer. We obtain a lower bound on the number of terminal sand piles by establishing a bijection between a subset of these sand piles and the set of shifted Young tableaux. We then prove that this number is at least factorial.
\end{abstract}

Keywords: Sand Pile Model, Young Tableaux

\section{Introduction}

The sand pile model was introduced by Bak, Tang and Wiesenfeld (see [BTW88]) in the context of selforganized criticality. Since then, there has been a lot of work on this model and related models, especially from the graph-theoretical point of view (see, e.g., [ALSSTW89, GK93, GMP]).

In the classical model, designed by Brylawski for another purpose (see [Bry73]), a sand pile is a decreasing sequence of integers $\left(n_{1} \geq \cdots \geq n_{k}\right)$. One can represent such a sand pile graphically by justifying at the bottom $k$ columns of respective lengths $n_{1}, \ldots, n_{k}$ (see Figure 1).

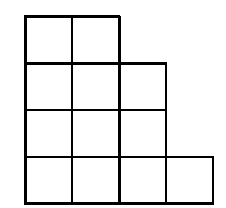

Figure 1 : The graphical representation of the sand pile $(4,4,3,1)$.

In the Brylawski model, there are two different transition rules, a vertical rule and a horizontal rule. In this paper, we will only consider the vertical one: a grain can move from one column to the next one on its right if the left column contains at least two grains more than the right one. For example, on the previous pile, one can only apply one transition between the third and the fourth column (see Figure 2). We will say that the third column topples.

1365-8050 (C) 2001 Maison de l'Informatique et des Mathématiques Discrètes (MIMD), Paris, France 


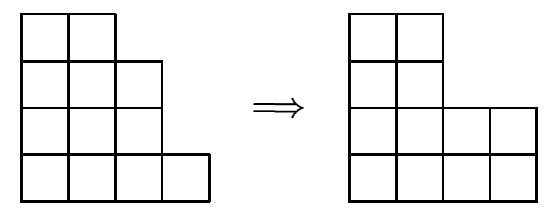

Figure 2 : The only transition for the pile $(4,4,3,1)$.

In our study, we add the hypothesis that all grains are distinct, that is : they are numbered with distinct integers. This new hypothesis makes it possible to study how the grains move and not only how the shape of the pile evolves.

This paper is structured as follows. We first recall the definition of the sand pile model SPM(0) and give two simple results about its topplings (Section 2). We then concentrate on characterizing final piles that can be transformed back into the initial pile (Section 3). Finally, in Section 4, we prove that the number of final piles is a factorial number, at least $\left(\frac{n}{2}\right)$ ! when the number $n$ of grains goes to infinity.

\section{Acknowledgements}

The authors would like to thank Michel Morvan for helpful discussions and Jacques Mazoyer and the project Parachute that allowed the authors to meet and work together in Departamento de Ingenería Matemática, Santiago, Chile.

\section{Basic definitions and remarks}

\subsection{Definitions and notations}

The Sand Pile Model we are going to study here is the model SPM(0). In this model, a grain can move from one column to the next one to the right if the left column contains at least two grains more than the right one. In our study, we add the hypothesis that all grains are distinct, that is: they are numbered with distinct integers. Starting with a single column $I(n)$ containing $n$ grains of sand stacked from top to bottom in increasing order by label, the natural question is to ask about all the completely toppled piles, that is, all the piles such that no grain can move and that can be obtained by toppling the initial one. With identical grains, the question is equivalent to find the shape of the final pile(s) and in this case, the answer is well-known and has been proved in many simple ways (see, e.g., [GK93, Pha99]) : the integer $n$ uniquely decomposes as a sum $\left(\begin{array}{l}p \\ 2\end{array}\right)+k$ where $p$ is the greatest possible integer and $k$ a non-negative integer. Then the shape of the final pile is, reading from left to right,

$$
(p-1, p-2, \ldots, k+1, k, k, k-1, \ldots 1) .
$$

With numbered grains, the final shape is obviously the same but it is obtained in many ways depending on the transitions.

For example, with $n=5$, one gets the transitions shown in Figure 3.

With $n=6$, one obtains for the first time more than one final pile and one can check that the two final piles are the ones shown in Figure 4.

In what follows, we denote by $T(n)$ the number of completely toppled piles. Here are the known values. 


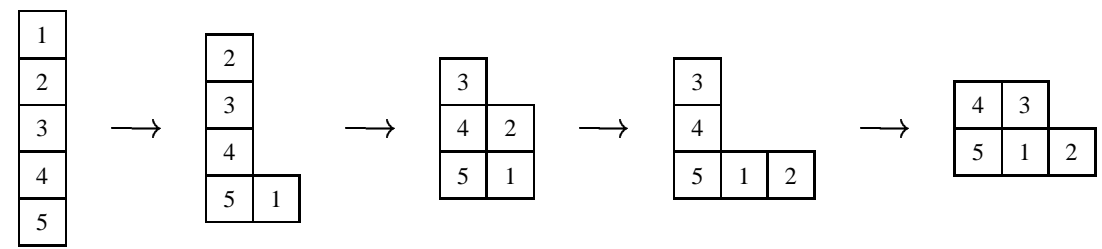

Figure 3 : The transitions with 5 grains.

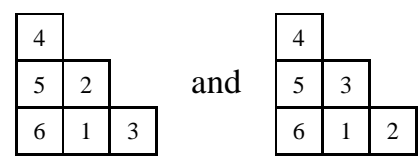

Figure 4 : The two final piles with 6 grains.

\subsection{Shifted Young tableaux}

In this subsection, we recall the definition of a special Young tableau, the so-called shifted Young tableau. It is a well-known combinatorial object, see, e.g., [Sag91].

Definition 2.1 Let $\lambda=\left(\lambda_{1}, \ldots, \lambda_{p}\right)$ be a strict partition of $n$, that is, a partition such that $\lambda_{1}>\lambda_{2}>\cdots>$ $\lambda_{p}$. One represents the associated shifted shape of $\lambda$ as a standard Young tableau in which the $i$-th row from the top has been shifted to the right by $i-1$ places so that the first column becomes a diagonal (see example below).

The shifted Young tableaux of shape $\lambda$ are all the fillings of its associated shifted shape with integers from 1 to $\mathrm{n}$ such that the integers increase in rows from left to right and in columns from top to bottom.

Example 2.2 Let $\lambda=(5,4,2,1)$. Then its shifted shape is

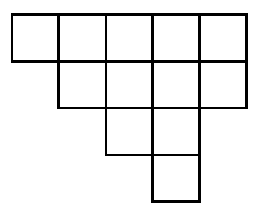

and an example of a shifted Young tableau of this shape is

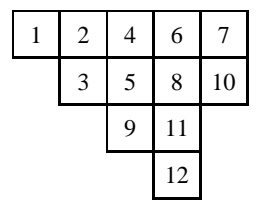

Remark 2.3 As for standard Young tableaux, there exists a hook-length formula that makes it possible to compute the number of shifted Young tableaux. If one represents the partition as a matrix, the shifted hook of a cell $(i, j)$ is 


\begin{tabular}{|l|l|l|l|l|l|l|l|l|l|l|l|l|l|l|l|}
\hline$n$ & 1 & 2 & 3 & 4 & 5 & 6 & 7 & 8 & 9 & 10 & 11 & 12 & 13 & 14 & 15 \\
\hline$T(n)$ & 1 & 1 & 1 & 1 & 1 & 2 & 2 & 3 & 6 & 21 & 21 & 47 & 123 & 438 & 2052 \\
\hline
\end{tabular}

\begin{tabular}{|l|l|l|l|l|l|}
\hline$n$ & 16 & 17 & 18 & 19 & 20 \\
\hline$T(n)$ & 2494 & 6741 & 25052 & 107343 & 525484 \\
\hline
\end{tabular}

Figure 5 : Exact known values for $T(n)$.

$$
H_{i, j}=\left\{\left(i^{\prime}, j\right), i^{\prime} \geq i\right\} \cup\left\{\left(i, j^{\prime}\right), j^{\prime} \geq j\right\} \cup\left\{\left(j+1, j^{\prime}\right), j^{\prime} \geq j+1\right\} .
$$

For example, the hook of the cell $(1,2)$ in the shifted shape $(5,4,3,1)$ is represented by the black cells in Figure 6.

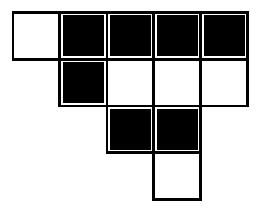

Figure 6 : The hook of $(1,2)$.

As for the number of standard Young tableaux, the number of shifted Young tableaux of shape $\lambda$ is given by the following formula:

$$
f^{\lambda}=\frac{n !}{\prod_{(i, j) \in \lambda} h_{i, j}}
$$

where $h_{i, j}$ is the cardinality of $H_{i, j}$.

\subsection{First remarks}

In this subsection, we provide the first remarks on the model we study. The proofs are very simple but will illustrate the other proofs since they contain the main simple ideas of the paper.

Remark 2.4 If one computes the ratios $T(n+1) / T(n)$, one can see that it is locally minimum when $n$ is a binomial coefficient $n=\left(\begin{array}{l}p \\ 2\end{array}\right)$ and maximum when $n=\left(\begin{array}{l}p \\ 2\end{array}\right)-1$ (see Figure 5). One can get the following interpretation for this property: the topplings behave as if there was a sort of diagonal toppling where all the grains of the same diagonal are able to permute. If we add one grain when there only misses one grain on a diagonal, then the growth ratio is maximal. Conversely, if we add the first grain of a new diagonal, then the ratio is minimal.

Remark 2.5 The previous remark shows in particular that it should always be useful to consider the piles as a union of diagonals and not as a union of columns or rows.

Remark 2.6 The sequence $(T(n))_{n \in \mathbb{N}^{*}}$ is an increasing sequence. 
Proof - Let $n$ be an integer. We then consider the $T(n)$ toppled piles with $n$ grains. For each of these piles, construct a sequence of topplings that starts with the initial pile and finishes on this pile. Put grain $n+1$ under the first column and repeat the toppling sequence; grain $n+1$ never moves and all the other grains end up in the same place as if grain $n+1$ had never existed. It is then obvious to see (for example, by induction on the number of topplings) that the same sequence of topplings starting with a column with $n+1$ grains leads to the same final pile, except that the first column contains one more grain. This proves at the same time that the transitions of the pile with $n+1$ grains are valid and that the piles obtained starting with $n+1$ grains are all distinct, so that $T(n+1) \geq T(n)$.

\section{Toppling and shifted Young tableaux}

In this section, we concentrate on the following problem: find generic elements that belong to the list of the completely toppled piles. In fact, the objective is to provide a complete characterization of the toppled piles but since this objective seems out of reach at present, we provide a set of elements that provably belong to the final list. These elements are the elements that are the easiest to "reverse" in a special sense. We will see in Section 4 another set of elements that belong to the final list but this result makes a nice relation between sand piles and Young tableaux. These two sets have the same asymptotic behavior but they clarify two differents parts of the topplings: the Young tableaux are well-known and this property allows an interesting connection with a usual combinatorial object; the diagonal elements that will be presented in Section 4 show the importance of diagonals in the topplings.

As one can very easily see with a few examples,for a given number $n$ of grains, the first column of any final pile is always the same and contains the elements from $n$ to $n-p+2$, reading it from bottom to top. So we can forget about this column and concentrate on the others.

Notation 3.1 Let $P$ be a pile. Let us denote by $C(P)$ and $R(P)$ respectively the first column of $P$ and the pile obtained by removing the first column of $P$.

Definition 3.2 Let $n$ be an integer of the form $\left(\begin{array}{l}p \\ 2\end{array}\right)$ and $P$ be a pile of shape $(p-1, p-2, \ldots, 2,1)$. We say that $P$ is easily invertible if

- $C(P)$ is filled with the integers from $n$ to $n-p+2$,

- For all integer $k$ between 1 and $n-p+1$, the grains numbered from 1 to $k-1$ form a shape with no interior hole and with columns of strictly decreasing length from left to right.

For example, the pile

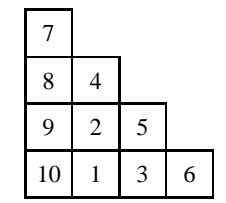

is easily invertible since the integers from 1 to $k$ have no two columns of the same length for all $k$ from 1 to 6.

This definition makes sense in the following way: if $P$ is an easily invertible pile, then the grain numbered $n-p+1$ can move back to the first column and then, the grain numbered $n-p$ and so on to the 
grain numbered 1: the condition that two columns never have the same length is equivalent to saying that the grain can move back from column $k$ to column 1 applying back $k-1$ times the vertical rule.

Theorem 3.3 Let $P$ be a pile of shape $(p-1, \ldots, 2,1)$. Then $P$ is an easily invertible pile if and only if

- $C(P)$ is filled with the integers from $n$ to $n-p+2$,

- $R(P)$ forms a Young tableau with the extra condition that the integers in the respective diagonals from the upper left corner to the lower right corner increase from left to right.

Proof - We prove the "if" and "only if" implications one at a time. Let us first assume that $P$ is easily invertible. Then the first condition of the theorem is satisfied. Moreover, the other columns form a Young tableau since no interior hole is created by removing the greatest entries (that is equivalent to say that the greatest entries are at the top of the columns or to the right of the rows, that is a possible definition of Young tableaux). Moreover, if a diagonal does not increase from left to right, let $y$ and $x$ be two consecutive elements of this diagonal such that $y>x$ and $y$ is to the left of $x$. Then, removing all the grains greater than or equal to $y$ leaves two columns of the same length, and contrary to the definition. So $P$ satisfies the conditions of the theorem.

Let us now assume that $P$ satisfies the conditions of the theorem. Then the first condition of the definition is satisfied. Moreover, let $k$ be an integer and consider the remaining pile after one has removed the grains with a value strictly greater than $k$. This pile has no interior hole thanks to the Young tableau condition. Finally, if $x$ is at the top of a non-empty column, then it has no element to its right thanks to the extra condition about diagonals (an element $y$ to its right must smaller than or equal to $k$ or it would have been removed; so the element which was on top of $x$ is less than $y$ and, therefore, less than $k$ and wouldn't have been removed). So all columns have a different length. Finally, $P$ is easily invertible.

Lemma 3.4 Let $A(n)$ be the set of piles with $n=\left(\begin{array}{l}p \\ 2\end{array}\right)$ grains that satisfy the conditions of the previous theorem. Then $A(n)$ is in bijection with the set of shifted Young tableaux of shape $(p-2, p-3, \ldots, 1)$.

Proof - We only describe the bijection since the proof immediately follows. Let $P$ be a pile that satisfies the conditions.

(i) Start from $P$ and construct $R(P)$.

(ii) Make a translation of all the columns of $R(P)$ so that they are top-justified and no longer bottomjustified.

(iii) Make a clockwise rotation of angle $\frac{\pi}{2}$ of the previous diagram.

Then the obtained diagram is a shifted Young tableau. One can easily see that the reverse algorithm exists and transforms a shifted Young tableau into an easily invertible pile.

Example 3.5 In Figure 7, there is an invertible pile and its image under this bijection - a shifted Young tableau.

Putting all things together, one gets the following result: 


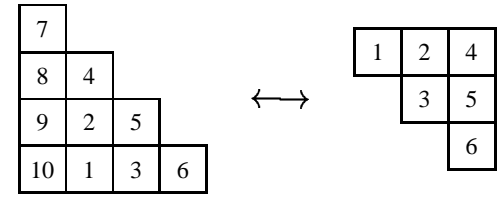

Figure 7 : An easily invertible pile and its image under the bijection

Corollary 3.6 The number of easily invertible piles is

where $\lambda$ is the partition $(p-2, p-3, \ldots, 1)$.

$$
\frac{\left(\begin{array}{c}
p-1 \\
2
\end{array}\right) !}{\prod_{(i, j) \in \lambda} h_{i, j}}
$$

Remark 3.7 One can easily extend the previous definition to all the final shapes and not only the staircase ones with the same conditions but written in a much more complicated way. Then all the theory is the same, the theorem remains true and so does the conclusion.

\section{Asymptotic behavior}

In this section, we provide a lower bound for the number of toppled piles. This lower bound is $\left(\frac{n}{2}\right) !$ and that proves that the number of toppled piles grows as a factorial when $n$ grows. To prove this result, we are going to show that a very simple set of piles belongs to the set of toppled piles. Let us first fix the notation and definitions. Notice that one can get the same asymptotic behavior using the shifted Young tableaux but this construction seems to us much simpler and can lead to further generalizations.

Notation 4.1 If $P$ is a pile, we will use the decomposition of $P$ as $\left(C(P), D(P), R^{\prime}(P)\right)$ where $C(P)$ is the first column of $P, D(P)$ is the top-most diagonal of $R(P)$ and $R^{\prime}(P)$ the remaining part of $R(P)$.

For example, in the following pile, $C(P)$ contains the integers from 7 to $10, D(P)$ contains the integers from 4 to 6 , and $R^{\prime}(P)$ contains the integers from 1 to 3 .

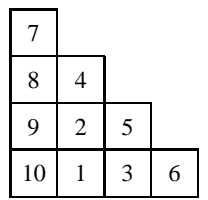

Definition 4.2 Let $n$ be an integer that decomposes as $n=\left(\begin{array}{l}p \\ 2\end{array}\right)+k$ with $0 \leq k<p$. Let $E(n)$ be the following set of piles:

- If $k=0$, the set $E(n)$ is the set of all toppled piles with $n$ grains.

- $E\left(\left(\begin{array}{l}p \\ 2\end{array}\right)+k\right)$ is the set of piles $P$ of shape $(p-1, p-2, \ldots, k+1, k, k, k-1, \ldots 1)$ such that

(i) $C(P)$ contains from top to bottom the integers from $\left(\begin{array}{c}p-1 \\ 2\end{array}\right)+k+1$ to $\left(\begin{array}{l}p \\ 2\end{array}\right)+k$, 
(ii) $D(P)$ has element $\left(\begin{array}{c}p-1 \\ 2\end{array}\right)+k$ at its top and a permutation of the integers from $\left(\begin{array}{c}p-1 \\ 2\end{array}\right)+1$ to $\left(\begin{array}{c}p-1 \\ 2\end{array}\right)+k-1$ in the other cells.

(iii) $R^{\prime}(P)$ is equal to a pile $R(Q)$ with $Q$ belonging to $E\left(\left(\begin{array}{l}p \\ 2\end{array}\right)\right)$.

For example, the set $E(9)$ is represented Figure 8.
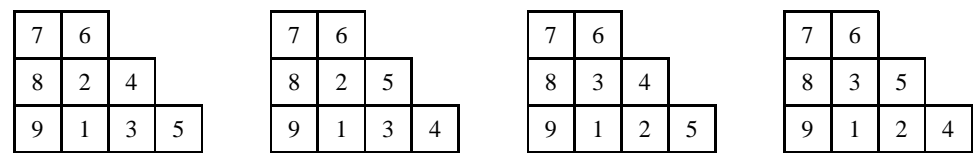

Figure 8 : The set $E(9)$.

We can now state our main theorem:

Theorem 4.3 For all integer $n$, the elements of $E(n)$ can be obtained by toppling the initial pile.

Proof - Let $n$ be an integer that can be written as $n=\left(\begin{array}{c}p \\ 2\end{array}\right)+k$. Let $P$ be an element of $E(n)$. We recall that $I(l)$ is the initial pile with $l$ grains.

Since it is possible to topple the pile $I\left(\left(\begin{array}{l}p \\ 2\end{array}\right)\right)$ to get columns as $R^{\prime}(P)$ (except the first one, see item (iii) of Definition 4.2) and doing as in the proof of Remark 2.6, it is also possible to partly topple $I(n)$ and obtain the integers from $\left(\begin{array}{c}p-1 \\ 2\end{array}\right)+1$ to $\left(\begin{array}{c}p \\ 2\end{array}\right)+k$ in the first column and the others as $R^{\prime}(P)$.

Let us denote by $X$ the corresponding pile. We are going to show that one can topple $X$ and get $D(P)$. This part consists in an insertion sorting algorithm. Since it is not so easy, we suggest to the reader to look in the same time at the example below. First, put the top-most element of the first column in the second one. Next, the process is always the same: having put $l$ elements from $\left(\begin{array}{c}p-1 \\ 2\end{array}\right)+1$ to $\left(\begin{array}{c}p-1 \\ 2\end{array}\right)+l$ in their right order in $D(P)$ and in the first columns of $X$, consider the new top-most element $z$ of the first column. First, shift from bottom to top the elements that are to its right in $D(P)$, then move $z$ until it stops. When this process finishes, move all the diagonal elements to their final positions (from bottom to top).

Example 4.4 Let us consider $n=14$ and try to build the diagonal $(10,7,9,8)$. Begin with the pile (denoted as $X$ in the previous proof):

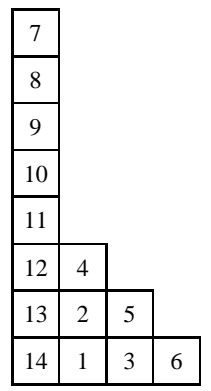

First, we put 7 in the second column. 


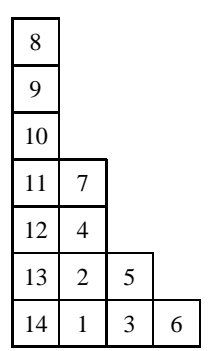

Next, since 8 is to the right of 7 in the final diagonal, 7 does not move and 8 moves (two times).

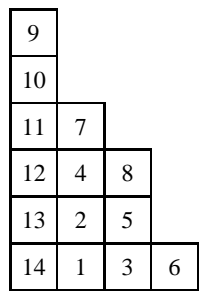

Next, since 8 is to the right of 9 in the final diagonal, 8 moves to the next column and then 9 moves (two times).

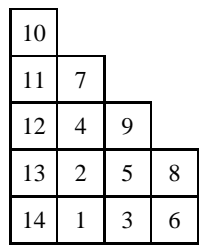

Finally, one moves each element of the last diagonal to their next column and moves 10 once.

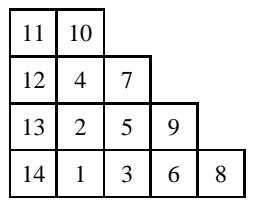

Corollary 4.5 Let $n$ be an integer that decomposes as $n=\left(\begin{array}{l}p \\ 2\end{array}\right)+k$ with $0<k<p$. Then one has:

$$
\operatorname{Card}(E(n))=(k-1) ! \operatorname{Card}\left(E\left(\left(\begin{array}{l}
p \\
2
\end{array}\right)\right)\right) .
$$

Remark 4.6 If $n$ is an integer such that $n=\left(\begin{array}{c}p+1 \\ 2\end{array}\right)$, then, doing as in the proof of Theorem 4.3, one sees that $E(n)$ in included in the set of piles such that: 
(i) $C(P)$ contains from top to bottom the integers from $\left(\begin{array}{c}p \\ 2\end{array}\right)+1$ to $\left(\begin{array}{c}p+1 \\ 2\end{array}\right)$,

(ii) $D(P)$ is a permutation of the integers from $\left(\begin{array}{c}p-1 \\ 2\end{array}\right)+1$ to $\left(\begin{array}{l}p \\ 2\end{array}\right)$.

(iii) $R^{\prime}(P)$ is equal to some $R(Q)$ with $Q$ belonging to $E\left(\left(\begin{array}{l}p \\ 2\end{array}\right)\right)$.

The following corollaries are easily deduced from the last corollary and the last remark.

Corollary 4.7 Let $n$ be an integer that decomposes as $n=\left(\begin{array}{c}p \\ 2\end{array}\right)+k$, with $0 \leq k<p$. Then one has:

$$
\operatorname{Card}(E(n)) \geq(k-1) ! \prod_{j=1}^{p-2} j !,
$$

with the convention that $(-1) !=0$.

Corollary 4.8 Let $n$ be an integer that decomposes as $n=\left(\begin{array}{l}p \\ 2\end{array}\right)+k$ with $0 \leq k<p$. Then one has:

$$
\operatorname{Card}(E(n)) \geq F(n)
$$

where $F(n) \sim\left(\frac{n}{2}\right)$ !

Proof - This proof can be done using the previous corollary and Stirling equivalent of $n$ !

\section{Conclusion}

This paper points out several key observations of a sand pile model known as $S P M(0)$ with numbered grains allowing a partial characterization on the final configurations. The minoration by $\left(\frac{n}{2}\right)$ ! is the only non-trivial known result and the experiments cannot help for guessing the true asymptotic behavior as even for $n=21$ the result seems rather huge for exact determination. As the Brylawsky model is an extension of this one, all our results remain true for it. Finally, there remains about this sand pile model many open problems such as determining the growth of the number of configurations and obtaining a complete characterization of those configurations.

\section{References}

[ALSSTW89] P. Anderson, L. Lovász, P. Shor, J. Spencer, E. TARdos and S. Winograd, Disks, ball, and walls: analysis of a combinatorial game, Amer. Math. Monthly, 96, 481-493, 1989.

[BTW88] P. BAK, C. TANG and K. Wiesenfeld, Self-organized criticality, Phys. Rev. A, 38, 364-374, 1988.

[Bry73] T. BRYLAWSKI, The lattice of integer partitions, Disc. Math., 6, 201-219, 1973.

[GK93] E. Goles and M. A. KIWI, Games on line graphs and sand piles, Theor. Comput Sci., 115, 321-349, 1993.

[GMP] E. Goles, M. MoRvan and H. D. Phan, Sandpiles and order strcture of integer partitions, to appear. 
[Pha99] H. D. Phan, PHD Thesis, Université Paris VII, 1999.

[Sag91] B. E. SAGAN, The Symmetric Group, Wadsworth and Brooks/Cole, California, 1991. 
